\title{
Assessment of suicidal behavior in dermatology (Review)
}

\author{
ANA MARIA ALEXANDRA STANESCU ${ }^{1}$, ALEXANDRA TOTAN ${ }^{1}$, DANIELA MIRCESCU $^{1}$, \\ SMARANDA DIACONESCU ${ }^{2}$, OVIDIU GABRIEL BRATU ${ }^{1,3}$, LÁSZLÓ FEKETE ${ }^{4}$, GYULA LÁSZLÓ FEKETE ${ }^{5}$, \\ DANIEL BODA $^{6}$ and CAMELIA CRISTINA DIACONU ${ }^{1,7}$
}

\author{
${ }^{1}$ University of Medicine and Pharmacy 'Carol Davila', 050474 Bucharest; ${ }^{2}$ II Pediatric Surgery Clinic, 'St. Mary' Children \\ Emergency Hospital, 700309 Iasi; ${ }^{3}$ Urology Department, Emergency University Central Military Hospital, 010825 Bucharest; \\ ${ }^{4}$ CMI Dermamed, 530540 Targu Mures; ${ }^{5}$ University of Medicine and Pharmacy, Dermatology Clinic, 530136 Targu Mures; \\ ${ }^{6}$ University of Medicine and Pharmacy 'Carol Davila', Department of Dermatology, 050474 Bucharest; \\ ${ }^{7}$ Clinical Emergency Hospital of Bucharest, 020322 Bucharest, Romania
}

Received September 20,2019; Accepted October 21, 2019

DOI: $10.3892 /$ etm.2019.8145

\begin{abstract}
Many diseases that affect physical health can also affect mental health. Many of these diseases are studied in terms of quality of life, depression and suicidal behavior. Dermatological disorders, although having a strong impact on the individual's life, are less studied with regard to suicidal behavior. There is a need to approach several dermatological diseases from the perspective of suicidal behavior in order to intervene early with specific treatment or to prevent suicide. Psoriasis, acne, melanoma, atopic dermatitis and urticaria are scarcely studied from the point of view of suicidal behavior. In addition to these diseases, there are others that require attention in terms of suicidal behavior, such as vitiligo or oculocutaneous albinism, most probably due to a small number of people suffering from these diseases, research is almost non-existent in these cases. We want to draw attention to the importance of early detection of suicidal behavior in dermatological practice and to highlight the need for several studies on this subject.
\end{abstract}

\section{Contents}

1. Introduction

2. Suicide in dermatology

3. Aspects of suicidal risk assessment in patients with dermatological diseases

4. Dermatological conditions with increased risk of suicidal behavior

5. Conclusions

Correspondence to: Professor Camelia Cristina Diaconu, University of Medicine and Pharmacy 'Carol Davila', 8 Eroii Sanitari Street, 050474 Bucharest, Romania

E-mail: drcameliadiaconu@gmail.com

Key words: suicidal behavior, dermatology, psoriasis, acne, melanoma

\section{Introduction}

The rate of psychological co-morbidity of dermatological disorders in Europe is not known, the patient being generally treated only dermatologically and in terms of other much more studied co-morbidities. In other diseases, such as diabetes, heart disease and asthma, the presence of depression is much more widely studied than in dermatological diseases.

The World Health Organization's slogan 'There is no health without mental health' highlights the fact that mental health contributes bidirectionally to physical health (1). On the one hand, mental health can affect physical health, but also physical health can affect mental health.

European studies have shown that patients with eczema, acne, psoriasis and hidradenitis suppurativa pose an increased risk of depression (2-4).

Although a less approached topic, suicide is encountered even in dermatology. The risk of suicide may be preexisting, may occur as a complication of skin disorders or can be triggered by drugs such as interferon. The risk of suicidal behavior can be an important factor in the general management of the dermatological patient. Patients at risk should be specifically asked about suicidal ideation and trends, with immediate intervention required (5). In dermatology, the risk of suicide has been described in severe congestive acne, psoriasis and metastatic melanoma (6). Patients with chronic disease or potentially fatal or severe pain may be suicidal, and patients with depression, alcohol dependence, substance abuse, schizophrenia or personality disorder are particularly at risk $(7,8)$. With regard to suicidal psycho-dermatological diseases, increased attention should be paid to the early recognition of a possible suicide risk (Fig. 1).

\section{Suicide in dermatology}

Suicide refers to the action of deliberately terminating one's own life; suicidal behavior can be classified as suicidal ideation, suicidal plan, and attempted suicide. Nonsuicidal self-harm refers to deliberate destruction of bodily tissue without suicidal intent, making it difficult to distinguish between non-suicidal self-injury and attempted suicide. 
Dieris-Hirche et al (9) conducted a study in Germany, showing that $16 \%$ of patients with atopic dermatitis had suicidal ideation, compared to the control group, of only $1 \%$.

In another study, Dalgard et al (10) observed a significantly higher prevalence of clinical depression in dermatological disorders, $10.1 \%$ vs. control $4.3 \%$, anxiety disorder $17.2 \%$ vs. control $11.1 \%$, and suicidal ideation $12.7 \%$ vs. control of $8.3 \%$.

Although many dermatological conditions do not endanger life, psychiatric disorders that may increase the risk of suicide should be considered due to the appearance of dermatological conditions that have a strong impact on the individual's life.

In patients with dermatological conditions, the factors associated with suicidal predisposition are psychiatric disorders, such as body dismorphous disorder, posttraumatic stress disorder and major depressive disorder. Suicide was associated with a psychiatric disorder in $90-95 \%$ of cases, and depression is a powerful predictor of suicidal ideation (11).

Certain dermatological symptoms (auto cut, auto-induced dermatosis) may be a sign of psychiatric disorders (major depressive disorder, posttraumatic stress disorder, personality disorder) (12).

Major stressful events and mental trauma can lead to exacerbation of dermatological conditions, increasing the risk of suicidal behavior (13).

Chronic severe conditions, such as psoriasis, are associated with suicidal ideation (independent of psychiatric comorbidity) (13). Also, pruritus syndrome correlates with severe depression, increasing the risk of suicidal behavior (14). Halvorsen et al (15) followed the suicidal idea in 4744 adolescents, $21.2 \%$ had suicidal ideation after intense pruritus, compared with $8.4 \%$ without pruritus.

Other chronic conditions associated with dermatological conditions, such as cancer, diabetes, cardiovascular disease, are alarm signals because patients have cumulative factors that predispose to suicide (16). Insomnia (or lack of sleep due to dermatological conditions) is another important factor to be considered in the suicidal risk assessment.

Regarding the layout of injuries, permanent or long-term injuries cause great problems, especially when these occur in childhood (12). Both facial and other lesions in the visible body or in the genital areas greatly affect the quality of life.

Medications used in dermatology, such as isotretinoin, tumor necrosis factor- $\alpha$ inhibitors, interleukin-17 (IL-17) inhibitors may have suicidal behavior as a side effect. On the other hand, the medication of psychiatric patients can cause dermatological conditions (multiforme erythema, severe seborrheic dermatitis, lithium-induced exacerbation of psoriasis or acne) (17).

Adalimumab has been associated with suicidal ideation, attempted suicide and complete suicide (18). Of the biological therapies, infliximab and adalimumab (tumor necrosis factor- $\alpha$ inhibitors) have the highest rate of suicidal behavior (19). Ustekinumab (targeting IL-12 and IL-23), secukinumab (anti-IL-17 agent) and ixekizumab (anti-IL-17 agent) were not associated with increased suicidal behavior. Brodalumab (an IL-17 receptor subunit A blocker) was initially banned because of the high rate of suicides following administration, however, later on it was concluded that there was lack of data regarding a clear association with suicidal behavior (20).
Regarding isotretinoin (retinoid), studies are contradictory. On the one hand, isotretinoin has been associated with aggression, psychosis, depressive behavior and suicide, on the other hand it has been associated with a significant improvement in quality of life and depression $(21,22)$. Monitoring depressive symptoms and suicidal thoughts in patients treated with isotretinoin should be observed, if they occur, the medication should be discontinued and a specialist counselor should be consulted (if necessary) (23). There have been reports of depression and suicidal ideation following the administration of retinitis etretinate and its metabolite acitretin $(24,25)$.

When doxycycline is administered, it may cause suicidal behavior and corticosteroids are associated with a wide range of neuropsychotic effects $(26,27)$.

\section{Aspects of suicidal risk assessment in patients with der- matological diseases}

As part of the suicide risk assessment of dermatological patients, age should be considered. In adolescents and young adults, the rate of suicide is rising, in middle-aged people there is a decline, then the rate of suicide increases in the elderly. The highest suicide rate worldwide is in people over 70 years of age and in the case of adolescents and young adults suicide is the second leading cause of death after traffic accidents (28). In the United States, the assessment of suicidal behavior in the $12-17$ age group showed that $15-29 \%$ have suicidal ideation, 12.6-19\% suicide plans, and 7.3-10.6\% attempted suicide (11).

Women have suicide attempts 3-4 times more often than men, but men are more likely to be successful in suicidal attempts. From another point of view, patients who are unemployed, lonely, divorced or having a deceased child/husband can have suicidal behavior.

Suicidal patients usually feel relieved when asked about suicide. Any threat of suicide is a psychiatric emergency. The risk of suicide should be considered in all patients with injuries caused by self-harm. In the case of suicidal thoughts, it is necessary to find out if there is a suicide plan and the necessary means. It is also necessary to take into account the personal or family history of suicidal behavior.

\section{Dermatological conditions with increased risk of sui- cidal behavior}

Psoriasis is a chronic disease, disfiguring, marked with remissions and exacerbations. Depending on the severity, the physical appearance may be extremely marked for the patient. In addition to the numerous comorbidities associated with psoriasis, there is also major depressive disorder. Of all dermatological disorders, psoriasis has the highest incidence of suicidal behavior (28). Gupta et al (29) found the desire for death to be present in $9.7 \%$ of patients with psoriasis studied, and 5.5\% had active suicidal ideation. Another study correlated the severity of psoriasis with depression and the suicidal ideation was present in $2.5 \%$ of patients with moderate psoriasis and $7.2 \%$ of patients with severe psoriasis (30). Numerous other studies have confirmed both the presence of depression and suicidal ideation in patients with psoriasis, and their association with the severity of psoriasis. Along with the increase in psoriasis severity, increased years 


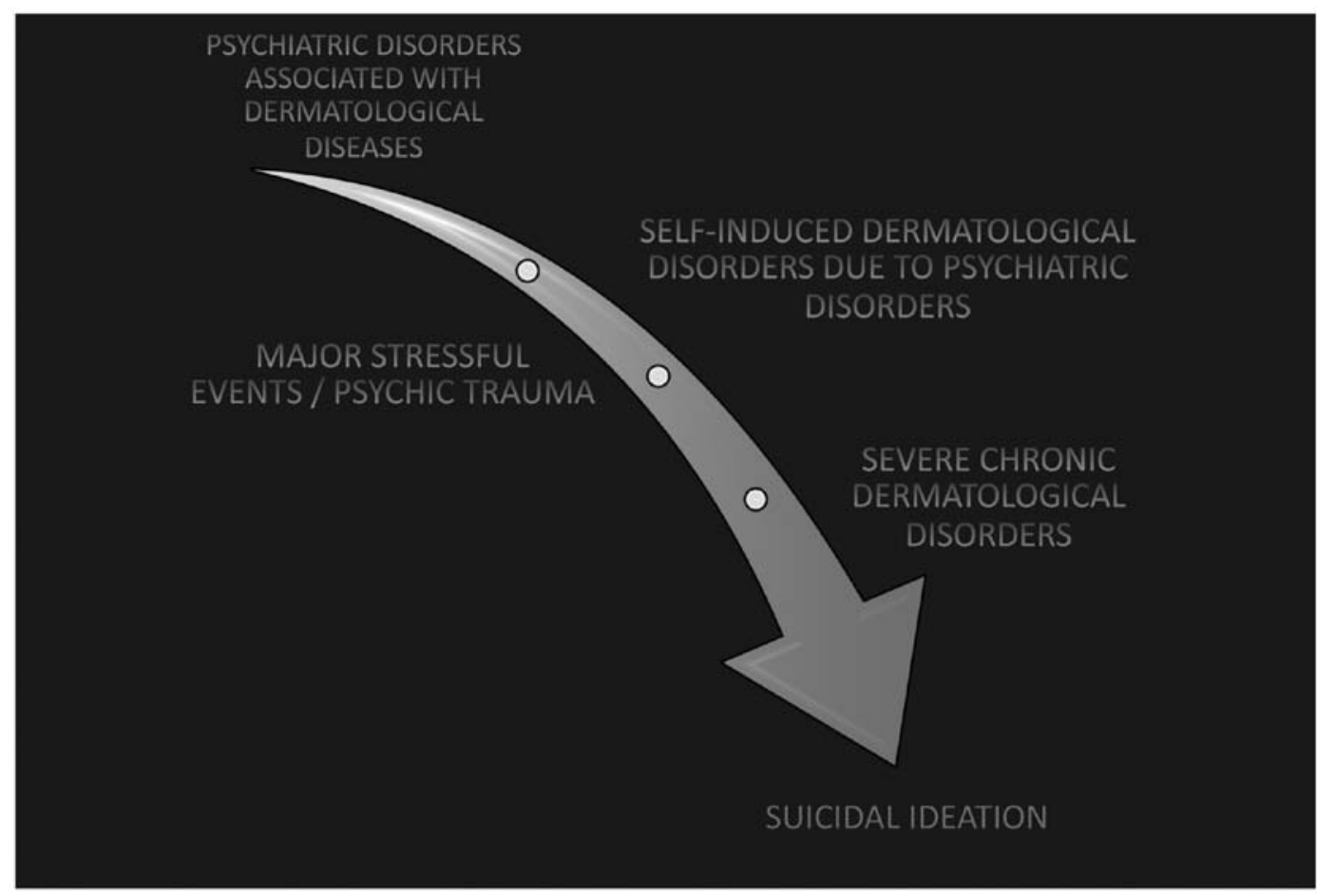

Figure 1. Influences of suicidal ideation.

of illness, poor treatment completeness, but also depending on the area of the affected body, increase the risk of suicidal behavior.

Acne affects mainly adolescents, with a higher degree of suicidal behavior $(31,32)$. Acne can be associated with isolation and difficulty in interpersonal relationships. The presence of scars, the shape and severity of acne contribute to suicidal behavior. A survey conducted in New Zealand on 9,567 young people reported the presence of suicidal ideation in $22.5 \%$ and attempted suicide in $5.2 \%$; interviewing only young people with acne, they reported $39.9 \%$ suicidal ideation and $12.9 \%$ attempted suicide (33). In women, more than in men, the presence of acne increases the risk of major depression, with major depression contributing to the suicide rate (34).

Melanoma is a challenge for both the doctor and the patient. Lu et al (35), in a cohort study in Sweden that included 12,669 people, recorded 22 suicides and 136 suicide attempts, with a relative risk of suicidal behavior after diagnosis of cancer of 1.6 (95\% CI, 1.4-1.9).

Atopic dermatitis falls into suicide risk dermatological diseases. In the spring, exacerbation of atopic conditions may increase the risk of suicide $(36,37)$. Halvorsen et al $(38)$ reported the presence of suicidal ideation in $15.5 \%$ of those studied with dermatitis vs. $9.1 \%$ of those who did not have dermatitis. A study in Japan reported the presence of suicidal ideation in the case of atopic dermatitis, in the mild form of $0.21 \%$, in the moderate form of $6 \%$ and in the severe form of $19.6 \%$, all three forms having a higher predisposition to female sex (39). In atopic dermatitis, pruritus and lack of sleep are correlated with depression. In addition to the patients with atopic dermatitis, the family also suffers, and parents of children with atopic dermatitis (depending on the severity of dermatitis) have thoughts of sacrificing their child or themselves reported as $0.11 \%$ in parents of children with mild dermatitis, $0.35 \%$ in parents of children with moderate dermatitis, and $3.28 \%$ in parents of children with severe dermatitis $(39,40)$.

Urticaria has a duration of less than $24 \mathrm{~h}$, but chronic urticaria lasts more than 6 weeks. Urticaria is a traumatic and stressful event, that can be associated with suicidal behavior. The suicidal ideation among patients with chronic urticaria was reported as $6.3-18.8 \%(41,42)$.

\section{Conclusions}

In practice, the psychological or psychiatric impairment of patients with dermatological conditions should not be neglected. Dermatological disorders are often associated with psychiatric disorders, requiring a specific approach to these patients by the dermatologist, and, if necessary, a multidisciplinary team need to be formed, including a psychiatrist. A complex approach is needed to estimate suicidal behavior. Suicidal behavior may be a psychiatric emergency, in some cases requiring immediate treatment.

\section{Acknowledgements}

Not applicable.

\section{Funding}

No funding was received.

\section{Availability of data and materials}

The datasets used and/or analyzed in the article are available from the corresponding author on reasonable request. 


\section{Authors' contributions}

AMAS, AT, DM, SD, LF and GLF collected, analyzed and interpreted the patient data regarding the risk of suicide in dermatology. AMAS, OGB, DB and CCD made substantial contributions to the conception of the study and interpretation of data; also, they drafted the manuscript and were major contributors in writing the manuscript. All authors read and approved the final manuscript.

\section{Ethics approval and consent to participate}

Not applicable.

\section{Patient consent for publication}

Not applicable.

\section{Competing interests}

The authors declare that they have no competing interests.

\section{References}

1. Prince M, Patel V, Saxena S, Maj M, Maselko J, Phillips MR and Rahman A: No health without mental health. Lancet 370: 859-877, 2007.

2. Cvetkovski RS, Zachariae R, Jensen H, Olsen J, Johansen JD and Agner T: Quality of life and depression in a population of occupational hand eczema patients. Contact Dermat 54: 106-111, 2006.

3. Dalgard F, Gieler U, Holm JO, Bjertness E and Hauser S: Self-esteem and body satisfaction among late adolescents with acne: Results from a population survey. J Am Acad Dermatol 59: 746-751, 2008

4. Onderdijk AJ, van der Zee HH,Esmann S, Lophaven S, Dufour DN, Jemec GB and Boer J: Depression in patients with hidradenitis suppurativa. J Eur Acad Dermatol Venereol 27: 473-478, 2013.

5. Harth W, Hillert A, Hermes B, Seikowski K, Niemeier V and Freudenmann RW: Suizidalität in der Dermatologie. Hautarzt 59: 289-296, 2008 (In German).

6. Singh S, Taylor C, Kornmehl H and Armstrong AW: Psoriasis and suicidality: A systematic review and meta-analysis. J Am Acad Dermatol 77: 425-440.e2, 2017.

7. Dieris-Hirche J, Gieler U, Petrak F, Milch W, Te Wildt B, Dieris B and Herpertz S: Suicidal ideation in adult patients with atopic dermatitis: A German cross-sectional study. Acta Derm Venereol 97: 1189-1195, 2017.

8. Tilea I, Petra D, Voidazan S, Ardeleanu E and Varga A: Treatment adherence among adult hypertensive patients: A cross-sectional retrospective study in primary care in Romania. Patient Prefer Adherence 12: 625-635, 2018.

9. Dieris-Hirche J, Gieler U, Kupfer JP and Milch WE: Suicidal ideation, anxiety and depression in adult patients with atopic dermatitis. Hautarzt 60: 641-646, 2009 (In German).

10. Dalgard FJ, Gieler U, Tomas-Aragones L, Lien L, Poot F, Jemec GB, Misery L, Szabo C, Linder D, Sampogna F, et al: The psychological burden of skin diseases: A cross-sectional multicenter study among dermatological out-patients in 13 European countries. J Invest Dermatol 135: 984-991, 2015.

11. Nock MK, Deming CA, Chiu WT, Hwang I, Angermeyer M, Borges G, Beautrais A, Viana MA, Karam EG, Kawakami N, et al: Mental disorders, comorbidity, and suicidal behavior. In: Suicide, Global Perspective from the WHO World Mental Health Surveys Nock MK, Borges G and Ono Y (eds). Cambridge University Press, New York, NY, pp148-163, 2012.

12. Cotterill JA and Cunliffe WJ: Suicide in dermatological patients. Br J Dermatol 137: 246-250, 1997.

13. Scott KM, Chiu WT and Hwang I: Chronic physical conditions and the onset of suicidal behavior. In: Suicide, Global Perspectives from the WHO World Mental Health Surveys. Nock MK, Borges G and Ono Y (eds). Cambridge University Press, New York, NY, pp164-178, 2012.
14. Gupta MA, Gupta AK, Schork NJ and Ellis CN: Depression modulates pruritus perception: A study of pruritus in psoriasis, atopic dermatitis, and chronic idiopathic urticaria. Psychosom Med 56: 36-40, 1994.

15. Halvorsen JA, Dalgard F, Thoresen M, Bjertness E and Lien L: Itch and pain in adolescents are associated with suicidal ideation: A population-based cross-sectional study. Acta Derm Venereol 92: 543-546, 2012.

16. Nicola P, Ardeleanu E, Gadau C, Dorobantu M, Darabont R, Tilea I, Varga A, Folescu R, Zamfir AS, Boanca M, et al: Evaluation of biochemical and clinical parametres of hypertension with type 2 Diabetes Mellitus. Rev Chim 69: 2402-2406, 2018.

17. Bliss SA and Warnock JK: Psychiatric medications: Adverse cutaneous drug reactions. Clin Dermatol 31: 101-109, 2013.

18. Ellard R, Ahmed A, Shah R and Bewley A: Suicide and depression in a patient with psoriasis receiving adalimumab: The role of the dermatologist. Clin Exp Dermatol 39: 624-627, 2014.

19. Eshuis EJ, Magnin KM, Stokkers PC, Bemelman WA and Bartelsman J: Suicide attempt in ulcerative colitis patient after 4 months of infliximab therapy - a case report. J Crohn's Colitis 4: 591-593, 2010.

20. Chiricozzi A, Romanelli M, Saraceno R and Torres T: No meaningful association between suicidal behavior and the use of IL-17A-neutralizing or IL-17RA-blocking agents. Expert Opin Drug Saf 15: 1653-1659, 2016.

21. GortonHC,WebbRT,KapurN and AshcroftDM:Non-psychotropic medication and risk of suicide or attempted suicide: A systematic review. BMJ Open 6: e009074, 2016.

22. Schrom K, Nagy T and Mostow E: Depression screening using health questionnaires in patients receiving oral isotretinoin for acne vulgaris. J Am Acad Dermatol 75: 237-239, 2016.

23. Rademaker M: Adverse effects of isotretinoin: A retrospective review of 1743 patients started on isotretinoin. Australas J Dermatol 51: 248-253, 2010.

24. Henderson CA and Highet AS: Depression induced by etretinate. BMJ 298: 964, 1989.

25. Arican O, Sasmaz S and Ozbulut O: Increased suicidal tendency in a case of psoriasis vulgaris under acitretin treatment. J Eur Acad Dermatol Venereol 20: 464-465, 2006.

26. Atigari OV, Hogan $C$ and Healy D: Doxycycline and suicidality. BMJ Case Rep 2013: pii: bcr2013200723, 2013.

27. Judd LL, Schettler PJ, Brown ES, Wolkowitz OM, Sternberg EM, Bender BG, Bulloch K, Cidlowski JA, de Kloet ER, Fardet L, et al: Adverse consequences of glucocorticoid medication: Psychological, cognitive, and behavioral effects. Am J Psychiatry 171: 1045-1051, 2014.

28. Naghavi M; Global Burden of Disease Self-Harm Collaborators: Global, regional, and national burden of suicide mortality 1990 to 2016: systematic analysis for the Global Burden of Disease Study 2016. BMJ 364: 194, 2019.

29. Gupta MA, Schork NJ, Gupta AK, Kirkby S and Ellis CN: Suicidal ideation in psoriasis. Int J Dermatol 32: 188-190, 1993.

30. Gupta MA and Gupta AK: Depression and suicidal ideation in dermatology patients with acne, alopecia areata, atopic dermatitis and psoriasis. Br J Dermatol 139: 846-850, 1998.

31. Nock MK, Borges G and Bromet EJ: The epidemiology of suicide and suicidal behavior. In: Suicide, Global Perspectives from the WHO World Mental Health Surveys. Nock MK, Borges G and Ono Y (eds). Cambridge University Press, New York, NY, pp5-32, 2012.

32. Grigore O, Mihailescu AI, Solomon I, Boda D and Caruntu C: Role of stress in modulation of skin neurogenic inflammation. Exp Ther Med 17: 997-1003, 2019.

33. Purvis D, Robinson E, Merry S and Watson P: Acne, anxiety, depression and suicide in teenagers: A cross-sectional survey of New Zealand secondary school students. J Paediatr Child Health 42: 793-796, 2006.

34. Yang YC, Tu HP, Hong CH, Chang WC, Fu HC, Ho JC, Chang WP, Chuang HY and Lee CH: Female gender and acne disease are jointly and independently associated with the risk of major depression and suicide: A national population-based study. BioMed Res Int 2014: 504279, 2014.

35. Lu D, Fall K, Sparén P, Ye W, Adami HO, Valdimarsdóttir U and Fang F: Suicide and suicide attempt after a cancer diagnosis among young individuals. Ann Oncol 24: 3112-3117, 2013.

36. Timonen M, Viilo K, Hakko H, Särkioja T, Meyer-Rochow VB, Väisänen E and Räsänen P: Is seasonality of suicides stronger in victims with hospital-treated atopic disorders? Psychiatry Res 126: 167-175, 2004. 
37. Solomon I, Ilie MA, Draghici C, Voiculescu VM, Căruntu C, Boda D and Zurac S: The impact of lifestyle factors on evolution of atopic dermatitis: An alternative approach. Exp Ther Med 17: 1078-1084, 2019.

38. Halvorsen JA, Lien L, Dalgard F, Bjertness E and Stern RS Suicidal ideation, mental health problems, and social function in adolescents with eczema: A population-based study. J Invest Dermatol 134: 1847-1854, 2014.

39. Kimata H: Prevalence of suicidal ideation in patients with atopic dermatitis. Suicide Life Threat Behav 36: 120-124, 2006.

40. Ilie MA, Caruntu C, Tampa M, Georgescu SR, Matei C, Negrei C, Ion RM, Constantin C, Neagu M and Boda D: Capsaicin: Physicochemical properties, cutaneous reactions and potential applications in painful and inflammatory conditions. Exp Ther Med 18: 916-925, 2019.
41. Zachariae R, Zachariae C, Ibsen HH, Mortensen JT and Wulf HC: Psychological symptoms and quality of life of dermatology outpatients and hospitalized dermatology patients. Acta Derm Venereol 84: 205-212, 2004.

42. Picardi A, Mazzotti E and Pasquini P: Prevalence and correlates of suicidal ideation among patients with skin disease. J Am Acad Dermatol 54: 420-426, 2006. 